\title{
Some Uses of Heat and Exercise in the Obstetric and Gynaecological Unit
}

\author{
(These treatment notes are based on extracts from a talk given by Dr. D. M. Lithgow, Specialist in Gynaecology at \\ Edenvale Non-European Hospital, who has kindly consented to allow them to be used in the Physiotherapy Journal.)
}

$\mathbf{P}^{\mathrm{h}}$ hysiotherapy is generally an adjunct to other methods of treatment in gynaecological cases and the two treatments which are most often called for are those which use heat, in all its forms, and exercises. Heat and exercises may be used singly, together or combined with other physio-therapeutic measures.

\section{PART I.}

- Heat Therapy covers a very wide field, and it is best considered under the differing headings of radiation, conduction, convection and conversion.

\section{Radiation.}

Radiant heat and infra-red rays fall under this heading, and are used for the mildly and frankly infected abdominal and perineal wounds in the maternity unit. In most hospitals both in South Africa and overseas, staphylococcal infections have proved a plague in the last few years. Now, at Edenvale with the use of a new and improved antiseptic (Hibitane) a stringently controlled central sterilising unit, a rigid scrub-up routine with Phisohex, and properly ventilated theatres, the number of these mildly infected wounds has lessened considerably.

\section{Conduction.}

Hot sitz baths, followed by half strength Eusol irrigations, are mainly used for the treatment of septic cases e.g. vesicovaginal fistulae and septic perineal wounds. After the septic condition has cleared, operations for the closure of the fistula or repair of the perineum are performed. Further physiotherapy is ordered later.

\section{Convection.}

A hot air cradle is of some value in cases of gynaecological backache in the acute stage.

Of the number of women attending hospital for treatment for bachache about $25 \%$ are caused by gynaecological conditions e.g. chronic cervicitis, parametritis, prolapse, endometriosis and chronic adnexal inflammations.

A backache with a genuinely gynaecological basis can be distinguished in many instances from the backache caused by a slipped disc or muscular rheumatism, by the great increase of pain at the menses.

\section{Conversion.}

Under this heading fall the diathermies-long wave, short wave and micro-wave. Without doubt this is the most useful weapon in the physiotherapists armoury as far as gynaecological work is concerned. Short wave diathermy is the method chiefly employed in the following examples:-

(i) Chronic Cervicitis which is one of the commonest troubles in the Non-European. It is often a cause of infertility as well as backache and discharge. Treatment with silver nitrate, cauterization, or conization and anti-biotics, is followed by short wave diathermy. In addition to the usual healing effects expected from short wave it seems to help the anti-biotics to permeate to the more deeply seated and infected cervical glands. (ii) Subacute and Chronic Salpingitis, i.e. inflammation of the tubes caused in $90 \%$ of Non-European cases by gonorrhoea, which in the female is a cruelly crippling and painful disease. Following an acute attack of Salpingitis the more chronic pyosalpinx and hydrosalpinx cases are seen. It is these cases which come for treatment with S.W.D.

(iii) Late radiation effects observed in those cases of cancer of the cervix which have been treated with deep X-Ray therapy and Radium can be ameliorated by further treatment with S.W.D.

(iv) Stricture of the rectum (Post lumphopathia venereum). Treated by short wave diathermy follows dilation with a set of Heygar's dilators.

(v) Post-partum Chronic Lymphoedema, termed by some people, phlegmasia alba dolens, responds fairly well to S.W.D., which is given after paravertebral sympathetic block, in conjunction with massage.

The extravagant claims made for the treatment of amenorrhoea, endocrine disorders, and dysmenorrhoea, by S.W.D. are not fully established. An occasional case, however, may respond quite dramatically to such treatment.

\section{Contra-indications to Short Wave Therapy.}

(i) The acute episodes in cases of tubal infection are not treated by S.W.D. The danger lies in causing a flare-up of symptoms. Large pysalpinges, in particular, are apt to rupture and death from septic shock may follow.

(ii) Cases of tuberculosis of the genital tract.

(iii) Menses.

(iv) Pregnacy.

(v) Endometriosis

N.B.- If the patient seems worse after treatment suspect genital Tuberculosis, or endometriosis, and refer back to the medical practitioner.

\section{Amount of treatment.}

Daily treatments by Short Wave Therapy are given for 2-3 wecks, and in bad cases as many as 18 treatments are rejuired. Anti-biotics and occasionally cortisone are used in addition. At Edenvale, surgery is employed following the Short Wave Diathermy course if palpable residual swellings remain after three weeks.

If the patient is a young woman a salpingostomy (opening up the ends of the tubes) is performed. With a woman in her forties a hysterectomy plus removal of the diseased adnexae is generally better, as it prevents the constant repetition of symptoms.

Ultra-Sonics, are not used at Edenvale Hospital for the treatment of gynaecological conditions, as it is possibly dangerous when used in the region of the ovaries. 


\section{WORLD CONFEDERATION FOR PHYSICAL THERAPY Ethical Principles for Physical Therapists}

The need for physical therapy is universal. The service of the physical therapist is, therefore, unrestricted
by considerations of nationality, race creed, colour, politics or social status.

The activities and relationships of physical therapists must vary widely in the many countries and spheres in which they are pursued and each national organisation must be free to make its own detailed code of ethics to which it may require its members to conform.

\section{Heat and Exercise-Continued from Page 7.}

\section{PART II.}

The second method of treatment favoured for gynaecological conditions is that of Exercise-here again divided into breathing exercises, assisted, free and resisted exercises.

\section{Breathing Exercises.}

Breathing exercises are given as a prophylactic measure against chest complaints following abdominal operations. In the Bantu respiratory troubles, particularly after Caesarean Section, seem to arise very quickly, and pre- and postoperative breathing exercises, together with leg exercises for the prevention of thrombosis, are of definite value.

If, in spite of these exercises, atelectasis occurs the use of a vibrator and back clapping will loosen secretions and promote healthier action of the lungs.

2. Assisted exercises.

In the treatment of post-natal dropped foot, -which is known by various names, from "obstetrical palsy" to "traumatic neuritis of the puerperium," -assisted exercises are used, following electrical stimulation by the current most suited to the condition. (In a very slight case, surging faradism will be tolerated, but in a bad case one of the progressive currents will be required.)

The Bantu patient on the whole has a smaller pelvis, from the obstetric capacity point of view, than the European. Consequently the pressure effect of the engaging head is more severe on the large lumbo-sacral nerve trunks which cross the alae of the sacrum. Damage to these nerve trunks, which form part of the great sciatic nerve, account for the comparatively large number of dropped foot cases seen in Non-European hospital practise.

\section{Free exercises.}

These include the well known ante- and post-natal exercises about which so much has already been written.

Dysmenorrhoea is greatly improved by free exercises. In similar cases among European women ballet lessons have proved helpful. Women suffering from this disability enjoy "free exercises."served in this fashion.

\section{Resisted exercises.}

The use of Kegels perineometer can be described as a "resisted exercise." Inserted vaginally it is used at Edenvale with marked success in causes of cystocele, rectocele and prolapse; and, in conjunction with faradism, in cases of stress incontinence, a lax pelvic floor, or for general toning of the levator ani in elderly hysterectomy cases.

Inserted rectally it can be used to improve ano-rectal tone and continence after cancer operations, where the removal of the bladder, uterus and vagina has become necessary.

In conclusion it can be truthfully said that with accurate diagnosis and well controlled therapy, great improvement in all types of gynaecological cases is possible by the use of heat and exercises.
The following general principles, to which all MemberOrganisations of the World Confederation for Physica! Therapy have declared their adherence, will serve as a guide to national organisations in building up their individual Code of Ethics:

1. Physical Therapists recognise not only the responsi bilities but also the limitations of their professional functions. They therefore only treat those patients referred to them by a registered medical practitioner.

2. Physical Therapists carry out with loyalty and the utmost skill any physical treatment prescribed by a registered medical practitioner which is aimed at restoring, improving or maintaining the mental and physical fitness of the patient.

3. Physical Therapists maintain at all times the highest standards of knowledge and skill.

4. Physical Therapists respect the culture and religious beliefs of their patients and associates.

5. Physical Therapists co-operate loyally with their physical therapy colleagues and with members of allied health professions and they sustain the confidence of the patient in all members of the health team.

6. Physical Therapists. hold in confidence all personal information entrusted to them and will nor discuss a patient's affairs with others than those responsible for the patient's care.

7. Physical Therapists recognise that failure of a colleague to conform to the ethical principles of the profession is detrimental to the patient and therefore such conduci should be reported to the proper authority. Unethical conduct should, however, never be reported in the presence of patients or associates.

8. Physical Therapists share responsibility with other citizens and members of other professions in meeting the needs of the public in matters of health, and should participate in all governmental and voluntary efforts to meet those needs.

9. Physical Therapists do not permit their names to be used in connection with the advertisement of products or with any other form of self-advertisement except as expressly authorised by the Code of Ethics of their national association.

10. Physical Therapists believe that just remuneration for their services should be received, but they may accept only such compensation or professional fee as the contract, actual or implied, provides.

11. Physical Therapists adhere at all times to standards of professional and personal ethics which reflect credit upon the profession. 